\title{
Dietary Zinc Intake and Kidney Stone Formation: Evaluation of NHANES III
}

\author{
Jie Tang $^{\mathrm{a}}$ Kim McFann $^{\mathrm{b}}$ Michel Chonchol ${ }^{\mathrm{a}}$ \\ a Division of Renal Disease and Hypertension, University of Colorado School of Medicine, Aurora, Colo., and \\ ${ }^{\mathrm{b}}$ Colorado School of Public Health, University of Colorado, Denver, Colo., USA
}

\author{
Key Words \\ Dietary zinc intake $\cdot$ Kidney stone disease $\cdot$ The Third \\ National Health and Nutrition Examination Survey
}

\begin{abstract}
Aims: We aimed to determine whether there is an association between dietary zinc intake (DZI) and prevalent kidney stone disease defined as self-report of any previous episode of kidney stone. Methods: We examined The Third National Health and Nutrition Examination Survey (NHANES III), a large US population-based cross-sectional study, and used logistic regression analyses to determine the independent association between DZI and prevalent kidney stone disease. Results: A total of 15,444 men and women over 18 years of age were eligible for analysis. Among them, 710 participants reported a history of kidney stones. Stone formers tended to have higher DZI than non-stone formers among NHANES III participants, though this difference did not reach statistical significance $(p=0.1)$. Multivariate adjusted logistic regression analysis revealed that higher DZI (log transformed) was associated with a significantly increased risk of kidney stone disease (odds ratio, OR $=1.41,95 \%$ confidence interval, $\mathrm{Cl}$ : 1.10-1.81, $\mathrm{p}=0.01$ ). After categorizing zinc intake into three groups, those with highest DZI (>15 mg/day) were also associated with a significantly increased risk of kidney stone disease, compared to those with lower DZI ( $<7 \mathrm{mg} /$ day; $\mathrm{OR}=1.70,95 \% \mathrm{Cl}: 1.13-2.57, \mathrm{p}=0.01)$. Conclusions: Our
\end{abstract}

study suggests that higher DZI is associated with increased risk of kidney stone disease. Future prospective studies are needed to clarify the causal relationship between zinc intake and kidney stone formation.

Copyright $\odot 2012$ S. Karger AG, Basel

\section{Introduction}

Kidney stone disease is common in the general population with an estimated prevalence of $10-15 \%$ in males and $3-5 \%$ in females [1]. It causes significant morbidity among those affected, and has a significant economic impact [2]. The risk of kidney stone varies with age, sex, lifestyle factors and geographical locations [1], and the majority ( $\sim 80 \%)$ of kidney stones are calcium based [3].

Trace elements are thought to affect the formation of calcium-based crystals [4]. Specifically, zinc is present almost exclusively in calcium-based stones [5], and has been shown to inhibit mineralization of rachitic cartilage in vitro [6]. Animal studies have also shown that zinc administration significantly reduced calcium and oxalate deposition in the renal papilla [7]. Atakan et al. [8] studied a total of 104 calcium oxalate stone formers and 77 normal controls, and found that stone formers had significantly lower serum and urine zinc levels compared to normal controls, indicating a possible inhibitory role of zinc on stone formation. However, other studies have

\section{KARGER}

Fax +4161306 1234

E-Mail karger@karger.ch

www.karger.com (c) 2012 S. Karger AG, Basel

0250-8095/12/0366-0549\$38.00/0

Accessible online at:

www.karger.com/ajn
Jie Tang, MD

Division of Renal Disease and Hypertension

University of Colorado School of Medicine

Aurora, CO 80045 (USA)

E-Mail Jie.tang@ucdenver.edu 
failed to support this finding $[9,10]$. Furthermore, a post hoc analysis of Age-Related Eye Disease Study showed that high zinc intake actually led to increased stone formation in men [11]. Thus far, all these previous human studies were limited by small sample size, and did not have adequate adjustment for significant confounders.

Since zinc is important for a variety of body processes, including immune regulation, cell metabolism, wound healing and appetite stimulation, dietary supplementation with zinc is currently a common practice. However, evidence is conflicting regarding whether higher body zinc store will increase risk of stone formation. In this analysis, we used a large US population survey database, the Third National Health and Nutrition Examination Survey (NHANES III), to examine the effect of higher dietary zinc intake (DZI) on the risk of kidney stone disease.

\section{Materials and Methods}

\section{Study Population}

NHANES III is a national probability sample of the total noninstitutionalized civilian population, 2 months of age or over in the United States. The survey collected demographic, socioeconomic, dietary, and health-related information, in addition to the examination and laboratory data obtained by highly trained medical personnel. The NHANES III is the first national survey to estimate total nutrient intake using food and beverage intake data from 24-hour recalls. In addition, daily supplement use was also recorded during household interviews.

There were a total of 33,994 participants in NHANES III, and our analyses were limited to 20,050 adult participants 18 years or older. Among those, 19,597 responded yes or no to the question regarding history of kidney stones. Responders who did not have complete information on dietary intake, had missing body mass index (BMI), serum calcium measurements, or had incomplete data for the history of hypertension, diabetes, and diuretic use, were excluded ( $n=4,153$ ). Thus, the final sample used in this study included 15,444 adult participants.

\section{Primary Predictor and Outcome}

The primary predictor was DZI. It was obtained by the estimates of zinc intake from food and beverages, using 24-hour dietary recall. It was collected by a trained dietary interviewer with the use of an automated, microcomputer-based interview and coding system. The type and amount of foods consumed were recorded with recall aids such as abstract food models, special charts, measuring cups, and rulers to help in quantifying the amounts consumed. Special probes were used to help the recall of commonly forgotten items such as condiments. DZI from food and beverages were calculated according to the food composition data from the US Department of Agriculture Survey Nutrient Database. Furthermore, participants and their proxy were asked about the use of vitamin and mineral supplements, the brand names of the products, and the frequency/dose used in the past month. The contribution of daily zinc intake from supplements was then calculated for each participant based on the zinc content of all products the person reported during the past month.

The outcome variable of interest was prevalent kidney stone disease. It was extracted from the interview data file. 'Have you ever had a kidney stone?' was the question asked during the standardized home interview. Adult participants who responded 'yes' to the question were considered to have a history of kidney stone in life.

\section{Covariates}

Age was defined as age at the time of the interview. Race/ethnicity was self-reported as non-Hispanic white, non-Hispanic black, Mexican American or other. History of hypertension was defined as self-reported physician diagnosis of hypertension. History of diabetes was defined as self-reported physician diagnosis of diabetes. BMI in kilograms per square meter was calculated from the weight and height measured during the physical examination. History of current hydrochlorothiazide (HCTZ) use was obtained during the standardized home interview. Dietary protein intake, calorie intake, salt intake and water intake were obtained from 24-hour dietary recall using the same methodology as mentioned for zinc intake. Total serum calcium concentration was measured using a NOVA $7+7$ electrolyte analyzer (Nova Biomedical, Waltham, Mass., USA).

\section{Statistical Analysis}

To be included in the study, participants had to have data on kidney stones, dietary zinc, protein, water and sodium intakes, age, sex, race, information regarding whether or not having a history of hypertension and diabetes, BMI, and serum calcium concentration. Models were also adjusted for the use of thiazide diuretics. Due to the complex sample strategy of NHANES III, appropriate 6-year weights and strata were applied. SAS (9.2) PROC SURVEYMEANS and SURVEYFREQ were used to obtain descriptive statistics for the population. Characteristics of the population were compared between stone formers and non-stone formers using the Rao-Scott $\chi^{2}$ for the categorical variables and ANOVA for the continuous variables. SAS (9.2) PROC SURVEYLOGISTIC was used to perform logistic regression to determine if DZI was associated with a history of kidney stones. Since DZI was not normally distributed and had some extreme outliers, a natural $\log$ transformation was applied. DZI was also divided into three categories using arbitrarily defined cutoffs $(<7,7-15$ and $>15 \mathrm{mg} /$ day), and comparisons were made between higher DZI group versus the lowest intake group. The $15 \mathrm{mg} /$ day upper limit used in this study was based on the 1989 recommended daily allowance. The lower limit of $7 \mathrm{mg} /$ day was arbitrarily assigned, which is $\sim 30 \%$ below the mean DZI (10.3 mg/day) in the general adult population (excluding pregnant and lactating women) reported by Briefel et al. [12]. Results are presented as odds ratio (OR) and $95 \%$ confidence interval (CI).

\section{Results}

There were a total of 15,444 participants eligible for the final analysis, including 710 who reported a history of kidney stone. Among stone formers, 120 (17\%) reported 
Table 1. Characteristics of the study population

\begin{tabular}{lccc}
\hline & $\begin{array}{l}\text { Stone } \\
\text { formers } \\
(\mathrm{n}=710)\end{array}$ & $\begin{array}{l}\text { Non-stone } \\
\text { formers } \\
(\mathrm{n}=14,734)\end{array}$ & $\begin{array}{l}\mathrm{p} \\
\text { value }\end{array}$ \\
\hline Age, years & $54 \pm 0.8$ & $43 \pm 0.2$ & $<0.0001$ \\
Men & $422(59.4)$ & $6,775(46)$ & $<0.0001$ \\
Race: non-Hispanic white & $607(85.5)$ & $9,956(67.6)$ & $<0.0001$ \\
History of hypertension & $283(39.9)$ & $3,813(25.9)$ & $<0.0001$ \\
History of diabetes & $79(6.4)$ & $1,150(7.81)$ & $<0.0001$ \\
BMI & $27.9 \pm 0.3$ & $26.3 \pm 0.1$ & $<0.0001$ \\
Dietary zinc, mg/day & $13.4 \pm 0.9$ & $12.0 \pm 0.1$ & 0.1 \\
Diuretic (HCTZ) use & $22(2.89)$ & $266(1.69)$ & $<0.01$ \\
Serum calcium, mg/dl & $9.3 \pm 0.02$ & $9.3 \pm 0.01$ & 0.6 \\
\hline
\end{tabular}

Values are expressed as means \pm SE or $n(\%)$.

Table 2. Differences in DZI by age categories

\begin{tabular}{lll}
\hline Age category & $\begin{array}{l}\text { Stone formers } \\
\mathrm{mg} / \text { day }\end{array}$ & $\begin{array}{l}\text { Non-stone formers } \\
\mathrm{mg} / \text { day }\end{array}$ \\
\hline $18-29$ years & $11.7 \pm 1.2$ & $12.7 \pm 0.2$ \\
$30-49$ years & $12.8 \pm 0.7$ & $12.4 \pm 0.2$ \\
$>50$ years & $13.9 \pm 1.5$ & $10.9 \pm 0.2$ \\
\hline
\end{tabular}

Values are expressed as means $\pm \mathrm{SE}$.

the use of zinc-containing supplements, as compared to 2,345 participants (16\%) among non-stone formers ( $\mathrm{p}=$ $0.4)$. As shown in table 1 , stone formers tended to be older, male, non-Hispanic white and had a higher BMI compared to non-stone formers. They were also more likely to have a history of hypertension, and had a higher usage of HCTZ diuretics. Stone formers had a mean DZI of 13.4 $\mathrm{mg} /$ day, compared to $12 \mathrm{mg}$ /day in non-stone formers (table 1). The difference was not statistically significant ( $\mathrm{p}=$ 0.1). Among stone formers, DZI increased with advancing age in both men and women (table 2), though it did not reach statistical significance $(\mathrm{p}=0.95)$. Overall, men had a significantly higher DZI than women, regardless of kidney stone history $(14.8 \pm 0.2$ vs. $9.6 \pm 0.1 \mathrm{mg} /$ day, $\mathrm{p}<$ 0.0001 ; table 3). Furthermore, since animal protein is one of the major dietary sources of zinc, we examined the relationship between DZI and protein intake, and found a significant positive correlation $(\mathrm{p}<0.0001)$.

After adjustment for age, sex, race, histories of diabetes and hypertension, BMI, dietary protein, water, salt and total calorie intake, usage of HCTZ and serum cal-
Table 3. Differences in DZI between men and women

\begin{tabular}{llll}
\hline & $\begin{array}{l}\text { Stone formers } \\
\text { mg/day }\end{array}$ & $\begin{array}{l}\text { Non-stone formers } \\
\text { mg/day }\end{array}$ & $\begin{array}{l}\text { Combined } \\
\text { mg/day }\end{array}$ \\
\hline Men & $\begin{array}{l}15.6 \pm 1.3 \\
(\mathrm{n}=422)\end{array}$ & $\begin{array}{l}14.7 \pm 0.2 \\
(\mathrm{n}=6,775)\end{array}$ & $\begin{array}{l}14.8 \pm 0.2 \\
(\mathrm{n}=7,197)\end{array}$ \\
\hline Women & $\begin{array}{l}10.3 \pm 1.0 \\
(\mathrm{n}=288)\end{array}$ & $\begin{array}{l}9.5 \pm 0.1 \\
(\mathrm{n}=7,959)\end{array}$ & $\begin{array}{l}9.6 \pm 0.1 \\
(\mathrm{n}=8,247)\end{array}$
\end{tabular}

Values are expressed as means \pm SE. DZI combined was higher in men than women $(\mathrm{p}<0.0001)$.

Table 4. Multivariate-adjusted OR of covariates from the model with categorized DZI

\begin{tabular}{lll}
\hline & OR $(95 \% \mathrm{CI})$ & p value \\
\hline Age & $1.03(1.02-1.03)$ & $<0.0001$ \\
Male & $1.70(1.34-2.15)$ & $<0.0001$ \\
Race: non-Hispanic white & $2.97(2.27-3.90)$ & $<0.0001$ \\
History of hypertension & $1.36(1.08-1.71)$ & $<0.01$ \\
History of diabetes & $1.11(0.79-1.58)$ & 0.5 \\
Protein intake & $0.996(0.99-1.001)$ & 0.12 \\
Sodium intake & $1.0(1.0-1.0)$ & 0.98 \\
Water intake & $1.0(1.0-1.0)$ & 0.46 \\
BMI & $1.03(1.01-1.05)$ & 0.002 \\
Diuretic $($ HCTZ) use & $1.34(0.69-2.60)$ & 0.4 \\
Serum calcium & $1.06(0.85-1.33)$ & 0.6 \\
Total calories & $1.00(0.999-1.00)$ & 0.1 \\
Zinc $7-15$ vs. $<7.0 \mathrm{mg}$ & $1.21(0.90-1.62)$ & 0.2 \\
Zinc $>15$ vs. $<7.0 \mathrm{mg}$ & $1.70(1.13-2.57)$ & 0.01 \\
\hline
\end{tabular}

cium concentration, higher DZI was significantly associated with prevalent kidney stone disease when zinc intake (log transformed) was modeled as a continuous variable $(\mathrm{OR}=1.41,95 \%$ CI: $1.10-1.81, \mathrm{p}=0.01)$. In addition, we evaluated kidney stone risk associated with extreme categories of DZI. We divided DZI into three categories. Among stone formers, there were 198 participants in category 1 ( $<7 \mathrm{mg} /$ day), 338 participants in category 2 (7-15 $\mathrm{mg} / \mathrm{d}$ ), and 174 participants in category 3 (>15 mg/day), whereas among non-stone formers, the corresponding numbers of participants were 4,519, 7,092 and 3,123 in each category. The multivariate-adjusted OR for stone formation was 1.70 (95\% CI: $1.13-2.57, \mathrm{p}=0.01)$ in those who consumed $>15 \mathrm{mg} /$ day zinc compared to those with $<7 \mathrm{mg}$ /day of DZI (table 4 ). Of note, this stone-promoting effect of DZI was again independent of total calorie intake. Unfortunately, among stone formers who reported 
supplement use, many did not have information regarding dose and quantity. Therefore, we were not able to include zinc supplement use in our final multivariate analyses.

There was no two-way interaction effect of age $\times$ zinc intake or of sex $\times$ zinc intake on kidney stone formation. Also, no three-way interaction effect of age $\times$ sex $\times$ zinc intake on kidney stone formation was noted. In our multivariate logistic regression analyses, the following variables were found to have significant associations with increased odds of prevalent kidney stone disease: older age, male sex, non-Hispanic white, history of hypertension, and increasing BMI. Higher protein intake was not associated with increased odds of prevalent stone disease.

\section{Discussion}

Zinc is important for a variety of body actions, and it is estimated that $14.6 \%$ of the US population were using at least one supplement containing zinc between 1988 and 1994 [12]. However, the effect of zinc use on kidney stone disease is unclear, and the results from existing studies have been contradictory. To our knowledge, this is the first study examining the independent association of higher DZI with the risk of kidney stone disease using a large United States population database.

We show that stone formers tend to have a higher DZI than non-stone formers. Higher DZI is associated with a significantly increased risk of prevalent kidney stone disease, especially when DZI exceeds $15 \mathrm{mg}$ /day. In addition, men in this study consumed significantly higher amounts of zinc from diet compared to women. This is consistent with the fact that men have a higher risk of prevalent and incident kidney stone disease compared to women $[1,13]$. Finally, DZI did not vary significantly by age in stone formers.

Our findings are consistent with what has been reported by Trinchieri et al. [9]. They showed that urinary zinc excretion was significantly higher in calcium stone formers than non-stone forming controls, suggesting a potential promoter role of zinc on calcium-based kidney stone formation. In addition, they found that men excreted more zinc than women, and the amount of urinary zinc excretion was independent of aging, consistent with our finding using DZI as a measure of body zinc store.

Our study does not provide an explanation for the finding. The role of heavy metals in kidney stone formation is not well established. Some of these heavy metals may have an effect on the initial crystallization of stone components, while others affect crystal aggregation and stone growth [14-16]. Zinc accounts for the majority of the heavy metals in calcium-based kidney stones [5], and is present in both stone nucleus and the crust [17]. It has been suggested from correlative studies that zinc acts as an inhibitor of kidney stone formation in humans $[8,10]$. However, results from other studies contradict these findings. In addition to the findings of Trinchieri et al. [9] reviewed earlier, Meyer and Angino [4] and Munoz and Valiente [15] failed to observe an inhibitory effect of zinc addition on calcium oxalate crystal growth through in vitro experiments. It is possible that zinc replaces calcium in the crystal lattice due to similar charge and molecular size, and promotes phosphate salt deposition [5, 18]. Slojewski et al. [18] analyzed stone samples from 219 kidney stone formers, and found that stone zinc content was directly correlated with the amount of phosphate salts. It is unclear whether this stone-promoting effect occurs at a certain threshold of zinc concentration in urine.

High DZI could also be a result of high dietary protein intake because the major source of dietary zinc is from foods of animal origin. However, zinc effect on stone formation appears to be independent of protein intake. We demonstrated in this study a neutral effect of protein intake on the risk of prevalent kidney stone disease. High protein intake was thought to be a risk factor for kidney stone formation due to acid load and high urinary calcium excretion [19]. However, several large observational studies have failed to show a positive correlation between dietary protein intake and kidney stones [20, 21]. Interestingly, a short-term metabolic study found that a controlled diet replacing vegetables with animal protein as main calorie source lowered urinary oxalate excretion [22].

Several limitations need to be mentioned. First, in this study, we used dietary intake instead of plasma zinc concentration as an indicator of body zinc status. DZI is a major determinant of body zinc store in people with adequate gastrointestinal absorption. However, potential gastrointestinal malabsorption (especially among elderly) should be considered, as it may misclassify individuals into different categories of zinc intake. A variety of biochemical indicators of body zinc status have been studied, including plasma zinc concentration, but none have been proven useful so far $[23,24]$. As a result of this, serum zinc concentrations were no longer measured in NHANES III. Second, because this study is cross-sectional, the present analysis is limited in its ability to establish causal or temporal relationships between DZI and 
kidney stone formation. However, it is very unlikely that stone formers increase their zinc intake for secondary stone prevention as existing studies have conflicting results, and no clinical guidelines to date recommend zinc intake for stone prevention. Third, the prevalent kidney stone cases were self-reported, and some participants may have kidney stone disease without self-awareness or clinical diagnosis. This may have led to potential misclassification, and should be non-selective with regard to zinc intake. However, if this misclassification exists, the results should be biased toward null. Fourth, we were unable to evaluate the effects of higher DZI on urine stone risk profile since measurements of these variables in urine (including urine zinc measurement) were not performed in NHANES III. Fifth, we were not able to include zinc-containing supplements in our analyses. However, the use of supplements between stone formers and nonstone formers was not different. Hence, the final results are unlikely to be affected. Finally, we do not have infor- mation on stone composition, although $80 \%$ of kidney stones in the general population like NHANES are calcium based.

\section{Conclusions}

We demonstrate that higher DZI is associated with a significantly increased risk of kidney stone disease among NHANES III participants. We speculate that zinc may enhance phosphate salt deposition in stone, thus leading to stone growth. Future prospective studies are needed to confirm this finding and address underlying mechanisms.

\section{Disclosure Statement}

None of the authors declare a competing interest.

\section{References}

1 Stamatelou KK, Francis ME, Jones CA, et al: Time trends in reported prevalence of kidney stones in the United States: 1976-1994. Kidney Int 2003;63:1817-1823.

-2 Saigal CS, Joyce G, Timilsina AR: Direct and indirect costs of nephrolithiasis in an employed population: opportunity for disease management? Kidney Int 2005;68:18081814.

-3 Aruga S, Honma Y: Urolithiasis and calcium metabolism. Renal calcium excretion and urolithiasis (in Japanese). Clin Calcium 2011; 21:1465-1472.

4 Meyer JL, Angino EE: The role of trace metals in calcium urolithiasis. Invest Urol 1977; 14:347-350

5 Bazin D, Chevallier P, Matzen G, et al: Heavy elements in urinary stones. Urol Res 2007;35: 179-184.

6 Bird ED, Thomas WC Jr: Effect of various metals on mineralization in vitro. Proc Soc Exp Biol Med 1963;112:640-643.

7 Sakly R, Zarrouk K, Hedhili A, et al: Study of anti-lithogenic action of zinc sulfate in experimental lithiasis in the rat. Ann Urol (Paris) 1991;25:246-249.

8 Atakan IH, Kaplan M, Seren G, et al: Serum, urinary and stone zinc, iron, magnesium and copper levels in idiopathic calcium oxalate stone patients. Int Urol Nephrol 2007;39: 351-356.
-9 Trinchieri A, Mandressi A, Luongo P, et al: The influence of diet on urinary risk factors for stones in healthy subjects and idiopathic renal calcium stone formers. Br J Urol 1991; 67:230-236.

10 Francois B, Cahen R, Pascal B: Inhibitors of urinary stone formation in 40 recurrent stone formers. Br J Urol 1986;58:479-483.

-11 Johnson AR, Munoz A, Gottlieb JL, et al: High dose zinc increases hospital admissions due to genitourinary complications. J Urol 2007;177:639-643.

12 Briefel RR, Bialostosky K, Kennedy-Stephenson J, et al: Zinc intake of the U.S. population: findings from the third National Health and Nutrition Examination Survey, 1988-1994. J Nutr 2000;130(suppl 5):1367S1373 S.

13 Almby B, Meirik O, Schonebeck J: Incidence, morbidity and complications of renal and ureteral calculi in a well defined geographical area. Scand J Urol Nephrol 1975;9:249253.

14 Fleisch H: Inhibitors and promoters of stone formation. Kidney Int 1978;13:361-371.

15 Munoz JA, Valiente M: Effects of trace metals on the inhibition of calcium oxalate crystallization. Urol Res 2005;33:267-272.

16 Sutor DJ: Growth studies of calcium oxalate in the presence of various ions and compounds. Br J Urol 1969;41:171-178.

17 Durak I, Kilic Z, Sahin A, et al: Analysis of calcium, iron, copper and zinc contents of nucleus and crust parts of urinary calculi. Urol Res 1992;20:23-26.
18 Slojewski M, Czerny B, Safranow K, et al: Microelements in stones, urine, and hair of stone formers: a new key to the puzzle of lithogenesis? Biol Trace Elem Res 2010;137:301316.

19 Pak CY, Odvina CV, Pearle MS, et al: Effect of dietary modification on urinary stone risk factors. Kidney Int 2005;68:2264-2273.

20 Curhan GC, Willett WC, Knight EL, et al: Dietary factors and the risk of incident kidney stones in younger women: Nurses' Health Study II. Arch Intern Med 2004;164: 885-8891.

21 Curhan GC, Willett WC, Speizer FE, et al: Comparison of dietary calcium with supplemental calcium and other nutrients as factors affecting the risk for kidney stones in women. Ann Intern Med 1997;126:497-504.

-22 Breslau NA, Brinkley L, Hill KD, et al: Relationship of animal protein-rich diet to kidney stone formation and calcium metabolism. J Clin Endocrinol Metab 1988;66:140146.

23 Bales CW, DiSilvestro RA, Currie KL, et al: Marginal zinc deficiency in older adults: responsiveness of zinc status indicators. J Am Coll Nutr 1994;13:455-462.

24 Walsh CT, Sandstead HH, Prasad AS, et al: Zinc health effects and research priorities for the 1990s. Environ Health Perspect 1994;(suppl 2):5-46. 